This item was submitted to Loughborough's Research Repository by the author.

Items in Figshare are protected by copyright, with all rights reserved, unless otherwise indicated.

\title{
Shared-control for fully actuated linear mechanical systems
}

PLEASE CITE THE PUBLISHED VERSION

https://doi.org/10.1109/cdc.2013.6760625

PUBLISHER

(c) IEEE

VERSION

AM (Accepted Manuscript)

LICENCE

CC BY-NC-ND 4.0

REPOSITORY RECORD

Jiang, Jingjing, and Alessandro Astolfi. 2019. "Shared-control for Fully Actuated Linear Mechanical Systems". figshare. https://hdl.handle.net/2134/36758. 


\title{
Shared-Control for Fully Actuated Linear Mechanical Systems
}

\author{
Jingjing Jiang $^{1}$ and Alessandro Astolfi ${ }^{2}$
}

\begin{abstract}
This paper presents a shared-control algorithm for fully actuated, linear, mechanical systems. It is assumed that the position of the mechanical system is constrained by a set of linear inequalities. These model convex and with the addition of "logical variables" non-convex feasible sets. The shared-control action is implemented using an hysteresis-based switching strategy. Formal properties of the algorithm are established using a partial Lyapunov analysis. Simulation results on simple case studies illustrate the effectiveness of the proposed algorithm.
\end{abstract}

\section{INTRODUCTION}

Shared-control refers to the ability of sharing the control action between a human operator and a feedback controller. The human operator takes overall responsibility of the operation of the system, while the feedback controller operates only in case of emergency or if the system evolves toward critical conditions. Shared-control is therefore of significant importance in modern applications, since partially humanly operated systems are widely used. Typical applications of shared-control can be found in vehicle design [1], surgery training systems [3] and mobility assistance [4].

We consider an abstract version of the shared-control problem with the objective to derive quantifiable properties of an underlying system in which two controllers, a human-inthe-loop and a feedback control, are operating. The sharedclosed-loop system has to operate safely at all time and this, in the present context, is characterized by the fact that only certain areas of the state space are admissible. Safe operation is therefore described as a collision avoidance problem, in which the obstacles are given by regions of the state space which cannot be visited by the state of the closed-loop system.

A popular method to solve the obstacle avoidance problem is the so-called Virtual Force Field (VFF) method [5]. This method relies on the introduction of a potential field, which models the environment, and forces the trajectories to evolve along descent directions. The goal should therefore be a global minimizer of the potential field. This method is however not adequate for shared-control. On one hand, it suffers from well-known shortcomings, such as the existence of local minimizers and oscillatory descent trajectories (typically around obstacles or narrow passages [6]). These

\footnotetext{
${ }^{1} \mathrm{~J}$. Jiang is with the Department of Electrical and Electronic Engineering, Imperial College London, UK, E-mail: jingjing.jiang10@imperial.ac.uk

${ }^{2} \mathrm{~A}$. Astolfi is with the Dept. of Electrical and Electronic Engineering, Imperial College London, London, SW7 2AZ, UK and DICII, University of Roma "Tor Vergata", Via del Politecnico 1, 00133 Rome, Italy, E-mail: a.astolfieimperial.ac.uk
}

have been partly alleviated by the use of Lyapunov-like methods [2] [7], together with barrier Lyapunov functions [8]. In several practical cases, barrier functions, also known as barrier certificates, could be constructed using semidefinite programming [13]. On the other hand, the Barrier Lyapunov Function method prevents the state of the system from reaching the boundary of the admissible set. However, in several practical applications, such as shared-autonomous parking or wheelchair operation, the goal to be reached may be the boundary of the admissible set: the trajectory has to reach the boundary while satisfying a "safety" constraint.

The approach pursued in the paper to solve the obstacle avoidance problem, which models the abstract shared-control problem studied, is to observe that the underlying system controlled by the human-in-loop and by the feedback controller can be regarded as an input redundant system, see for example [9] and references therein, and that input allocation can be studied using the techniques developed in [11]. In particular, in [9], it has been shown that for input-redundant systems an auxiliary signal can be used to promote or penalize actuators without affecting the response of the system (in the so-called strongly redundant case) or the steadystate response (in the so-called weakly redundant case). An alternative solution to the allocation problem, based on optimization concepts, has been presented in [10]. The problem of uniting global and local controllers is similar to the allocation problem. In [11] this problem has been solved using the so-called composite controller, which coincides with a given local controller in a neighbourhood of the origin and with a globally stabilizing controller elsewhere. Finally, Lyapunov methods have also been used in [12] to design a scalar, continuous, function yielding a smooth transition from the human input to the control input whenever the state of the system moves out of the safe region.

This paper is organized as follows. In Section II, the problem under investigation is formalized and a few standing assumptions are given. A novel strategy to solve the shared-control problem is given in Section III, where formal properties of the closed-loop system are also established. Section IV contains a few simulation results illustrating the theoretical developments. Finally, Section V contains some concluding remarks and suggestions for future research.

Since in the paper an abstract version of the shared-control problem is studied, we model the input of the human operator as either a (possibly time-varying) feedback strategy, or as an open-loop strategy. 


\section{PROBLEM STATEMENT}

In this section we formulate the shared-control problem that is solved in the paper. Note that, for simplicity, the underlying system to be controlled is a linear, fully-actuated, mechanical system, although the same ideas can be used for more general classes of systems.

Consider a linear, fully-actuated, mechanical system the dynamics of which are described by the equation

$$
\mathcal{M} \ddot{p}+\mathcal{K} \dot{p}+\mathcal{G} p=u_{s},
$$

where $p(t) \in \mathbb{R}^{n}$ denotes the generalized position, $u_{s}(t) \in$ $\mathbb{R}^{n}$ denotes the external forces, $\mathcal{M}^{\prime}=\mathcal{M}>0$ is the constant inertia matrix, $\mathcal{K}^{\prime}=\mathcal{K} \geq 0$ models the constant Coulumb friction forces, and $\mathcal{G}^{\prime}=\mathcal{G}$ describes the potential forces.

Let

$$
u_{s}(p, \dot{p}, t)=[1-k(p, \dot{p})] u_{c}(p, \dot{p}, t)+k(p, \dot{p}) u_{h}(p, \dot{p}, t),
$$

where $u_{h}$ describes the human input action, $u_{c}$ represents the control input and $k \in[0,1]$ quantifies how the control action is shared. In what follows we use the name $h$-control to denote the human input action, the name c-control to denote the control action, possibly delivered by a feedback controller, the name s-control to denote the shared-control action, and the name sharing function to denote the function $k$. Furthermore, we use the name h-closed-loop system to denote the closed-loop system described by the equation

$$
\mathcal{M} \ddot{p}(t)+\mathcal{K} \dot{p}(t)+\mathcal{G} p(t)=u_{h},
$$

and the name s-closed-loop system to denote the closed-loop system described by equation (1) with $u_{s}$ as in equation (2). Finally, let $\Omega_{h}\left(\Omega_{s}\right.$, resp.) be the $\Omega$-limit set ${ }^{1}$ of the h-closedloop system (s-closed-loop system, resp.).

In what follows, with some minor loss of generality, we assume that the sets $\Omega_{h}$ and $\Omega_{s}$ are composed of isolated equilibria (or periodic trajectories). Note that this assumption is not unnatural from an application perspective.

Note that systems (1) and (3) share the same state space $\mathcal{X}=\mathcal{P} \times \mathcal{V}=\mathbb{R}^{n} \times \mathbb{R}^{n}$, where $\mathcal{P}$ is the set of configurations and $\mathcal{V}$ is the set of velocities of the mechanical system.

Let $\mathcal{P}_{a} \subset \mathcal{P}=\mathbb{R}^{n}$ be a given, closed, connected, set of achievable configurations ${ }^{2}$, with non-zero volume and $u_{h}(p, \dot{p}, t)$ be a given $\mathrm{h}$-control. The shared-control problem can be posed as follows.

Given the system (1) and the set $\mathcal{P}_{a}$ find (if possible)

- a safe set $\mathcal{R}_{s}=\mathcal{P}_{a} \times \mathcal{V}_{s} \subset \mathcal{P}_{a} \times \mathcal{V}=\mathcal{R}$;

\footnotetext{
${ }^{1}$ Recall that the $\Omega$-limit set is forward invariant.

${ }^{2}$ Note that the set $\mathcal{P}_{a}$ can be imposed by the user, or by regulatory bodies and it has a similar definition as the maximal output admissible set given in [15].
}

- a c-control $u_{c}$;

- a sharing function $k$;

such that the closed-loop system (1)-(2) has the following properties.

(P1) The set $\mathcal{R}$ is forward invariant for the s-closed-loop system, i.e. for all initial state $(p(0), \dot{p}(0)) \in \mathcal{R}$, the state $(p(t), \dot{p}(t))$ of the s-closed-loop system remains in $\mathcal{R}$ for all $t \geq 0$.

(P2) The set $\Omega_{s}$ is such that

$$
\Omega_{s}= \begin{cases}\Omega_{h} & \text { if } \Omega_{h} \subset \mathcal{R}_{s}, \\ \Pi_{\mathcal{R}_{s}}\left(\Omega_{h}\right) & \text { if } \Omega_{h} \not \subset \mathcal{R}_{s},\end{cases}
$$

where $\Pi_{\mathcal{R}_{s}}\left(\Omega_{h}\right)$ denotes the projection (to be defined) of $\Omega_{h}$ into $\mathcal{R}_{s}$.

The projection of a set $S$ into a closed, connected set $T$, with nonzero volume, can be defined as follows. Let $s$ be a point in $S$. The projection of $s$ into $T$, denoted $\Pi_{T}(s)$ is given by

$$
\Pi_{T}(s)= \begin{cases}s & \text { if } s \in T, \\ \arg \min \operatorname{dist}(s, T) & \text { if } s \notin T,\end{cases}
$$

where $\operatorname{dist}(s, T)$ denotes the distance (i.e. Euclidean norm) between the point $s$ and the set $T$. Then the projection of $S$ into $T$ is the set

$$
\Pi_{T}(S)=\left\{p \in T \mid p=\Pi_{T}(s), \forall s \in S\right\} .
$$

In what follows we assume that the set $\Pi_{R_{s}}\left(\Omega_{h}\right)$ has the same "structure" of $\Omega_{h}$, i.e. if $\Omega_{h}$ is a singleton then $\Pi_{R_{s}}\left(\Omega_{h}\right)$ is a singleton (if $\Omega_{h}$ is a closed curve then $\Pi_{R_{s}}\left(\Omega_{h}\right)$ is a closed curve).

\section{DESIGN OF THE S-CONTROL}

In this section we provide a solution to the problem stated in Section II for the case in which the set $\mathcal{P}_{a}$ is defined by means of a set of linear inequalities and $\Omega_{h}$ is a singleton.

To begin with, consider a state-space representation of system (1) given by the equations

$$
\left\{\begin{array}{l}
\dot{p}=v \\
\dot{v}=\mathcal{M}^{-1}\left(-\mathcal{K} v-\mathcal{G} p+u_{s}\right),
\end{array}\right.
$$

and assume that the region $\mathcal{P}_{a}$ is defined as

$$
\mathcal{P}_{a}=\left\{p \in \mathbb{R}^{n} \mid \mathcal{S} p+\mathcal{T} \leq 0\right\},
$$

where $\mathcal{S}=\left[s_{1}^{T}, s_{2}^{T}, \ldots, s_{m}^{T}\right]^{T} \in \mathbb{R}^{m \times n}$ and $\mathcal{T}=$ $\left[t_{1}, t_{2}, \ldots, t_{m}\right]^{T} \in \mathbb{R}^{m}$.

In addition, the equation describing the region $\mathcal{P}_{a}$ are such that the following assumption holds. 
Assumption 1: $\mathcal{S}$ and $\mathcal{T}$ in (5) are such that

$$
\operatorname{rank}\left(\left[\begin{array}{l}
s_{r_{1}} \\
\vdots \\
s_{r_{l}}
\end{array}\right]\right)<\operatorname{rank}\left(\left[\begin{array}{ll}
s_{r_{1}} & t_{r_{1}} \\
\vdots & \vdots \\
s_{r_{l}} & t_{r_{l}}
\end{array}\right]\right)
$$

for all $l \in[n+1, m]$, where $r_{1}, r_{2}, \ldots, r_{l} \in\{1,2, \ldots, m\}$.

This assumption is needed to rule out degenerate situations, as discussed in the following statements.

Lemma 1: Consider the $m$ constraints (5). Assume Assumption 1 holds. If the set of points satisfying the inequalities (5) is non-empty then it has non-zero volume.

Lemma 2: Consider the $m$ constraints (5). Assume Assumption 1 holds. Then for any specific $v$, no more than $n$ constraints are active ${ }^{3}$.

We conclude this section noting that, in the rest of the paper, we implicitly assume that Assumption 1 holds and that $\mathcal{P}_{a}$ is not empty.

\section{A. Design of the c-control}

In this section we design the c-control under the assumption that $m=n$. This is without loss of generality if $m<n$, since in this case it is always possible to add auxiliary constraints, described for example by equations of the form

$$
p_{i}-\overline{p_{i}} \leq 0, \quad \text { or } \quad-p_{i}+\underline{p_{i}} \leq 0,
$$

for some $i$, where $p_{i}$ is the $i^{\text {th }}$ element of the vector $p$ and $\overline{p_{i}}\left(\underline{p_{i}}\right.$, resp.) is a sufficiently large (small, resp.) constant, to make $m=n$.

If $m>n$, then the c-control is designed on the basis of only $n$ constraints. This implies that there are $N_{c}$ c-controls where the $i^{\text {th }}$ c-controller $u_{c}^{i}$ is designed for the $i^{\text {th }}$ group of $n$ active constraints. In general,

$$
N_{c} \leq\left(\begin{array}{c}
m \\
n
\end{array}\right)
$$

Consider the $i^{\text {th }}$ set of $n$ active constraints, described by the inequality

$$
\mathcal{S}^{i} p+\mathcal{T}^{i} \leq 0
$$

and define the new partial coordinates

$$
x^{i}=\mathcal{S}^{i} p+\mathcal{T}^{i} .
$$

Relative to such $i^{\text {th }}$ group of $n$ active constraints, the admissible set $\mathcal{R}$ can be partitioned into three subsets $\mathcal{R}_{s}^{i}, \mathcal{R}_{h}^{i}$ and

${ }^{3}$ For each velocity $v \in \mathbb{R}^{n}$, the $j^{\text {th }}$ constraint is active if

$$
\exists k>0: s_{j}(k v)+t_{j}=0 .
$$

$\mathcal{R}_{d}^{i}$ defined by equations (6) in the next page, where $\mathcal{X}_{a}^{i}=$ $\mathcal{S}^{i} \mathcal{P}_{a}+\mathcal{T}^{i}$, and $b_{2}>b_{1}>0$. Note that, by construction, $\mathcal{R}_{s}^{i} \cup \mathcal{R}_{t}^{i} \cup \mathcal{R}_{d}^{i}=\mathcal{X}_{a}^{i} \times \mathbb{R}^{n}$ for all $i \in\left\{1,2, \ldots, N_{c}\right\}$. In addition, since $\mathcal{R}_{d}^{i}$ and $\mathcal{R}_{d}^{j}$ relate to different $v$, by taking Lemma 2 into consideration, we conclude that the condition

$$
\mathcal{R}_{d}^{i} \cap \mathcal{R}_{d}^{j}=\emptyset
$$

holds, for all $i \neq j$. Recall that for each subset $\mathcal{R}_{d}^{i}$ one c-control is designed. The fact that the sets $\mathcal{R}_{d}^{i}$ are disjoint implies that there is no ambiguity on which c-control is active at any given time instant.

Using the partial coordinates $x^{i}$, the system with the $i^{\text {th }}$ c-control, together with the associated constraints, can be rewritten as

$$
\begin{aligned}
\dot{x}^{i} & =\mathcal{S}^{i} \dot{p}=\mathcal{S}^{i} v, \\
\dot{v} & =\mathcal{M}^{-1}\left(-\mathcal{K} v-\mathcal{G}\left(\mathcal{S}^{i}\right)^{-1}\left(x^{i}-\mathcal{T}^{i}\right)+u_{c}^{i}\right), \\
x^{i} & \leq 0 .
\end{aligned}
$$

Let $z^{i}=\left(z_{1}^{i}, z_{2}^{i}, \ldots, z_{n}^{i}\right)^{T}$, with

$$
z_{j}^{i}=\log \frac{x_{j}^{i}+\epsilon_{j}^{i}}{x_{r_{j}}^{i}+\epsilon_{r_{j}}^{i}},
$$

for all $j \in\{1, \ldots, n\}$, where the constant $x_{r_{j}}^{i}$ describes the desired behaviour of the state $x_{j}^{i}$, and

$$
\epsilon_{j}^{i}=\left\{\begin{array}{ll}
0 & \text { if } x_{j}^{i}<0, \\
<0 & \text { otherwise }
\end{array} \quad \epsilon_{r_{j}}^{i}= \begin{cases}0 & \text { if } x_{r_{j}}^{i}<0 \\
<0 & \text { otherwise }\end{cases}\right.
$$

Differentiating the variables $z^{i}$ and $v$ yields

$$
\begin{aligned}
\dot{z}^{i}= & \operatorname{diag}^{-1}\left(\left(x_{r_{1}}^{i}+\epsilon_{r_{1}}^{i}\right) e^{z_{1}^{i}}, \ldots,\left(x_{r_{n}}^{i}+\epsilon_{r_{n}}^{i}\right) e^{z_{n}^{i}}\right) \mathcal{S}^{i} v \\
\dot{v}= & \mathcal{M}^{-1}\left[-\mathcal{K} v-\mathcal{G}\left(\mathcal{S}^{i}\right)^{-1}\left(\left(x_{r}^{i}+\epsilon_{r}^{i}\right) e^{z^{i}}-\epsilon^{i}-\mathcal{T}^{i}\right)\right] \\
& +\mathcal{M}^{-1} u_{c}^{i}
\end{aligned}
$$

where we have used the notation

$\left(x_{r}^{i}+\epsilon_{r}^{i}\right) e^{z^{i}}-\epsilon^{i}=\left[\left(x_{r_{1}}^{i}+\epsilon_{r_{1}}^{i}\right) e^{z_{1}^{i}}-\epsilon_{1}^{i} ; \ldots ;\left(x_{r_{n}}^{i}+\epsilon_{r_{n}}^{i}\right) e^{z_{n}^{i}}-\epsilon_{n}^{i}\right]$.

Let $v^{i *}=\left(v_{1}^{i *}, \ldots, v_{n}^{i *}\right)^{T}$ be the solution of the equations

$$
s_{j}^{i} v^{i *}=-z_{j}^{i}\left(x_{r_{j}}^{i}+\epsilon_{r_{j}}^{i}\right),
$$

for all $j \in\{1,2, \ldots, n\}$, where the vectors $s_{j}^{i}$ are such that $\mathcal{S}^{i}=\left[s_{1}^{i^{T}}, s_{2}^{i^{T}}, \ldots, s_{n}^{i^{T}}\right]^{T}$. Note that there always exists a solution to (10) since $\mathcal{S}^{i}$ is full rank.

Finally, consider the Lyapunov function

$$
L^{i}\left(z^{i}, v\right)=\frac{1}{2}\left[z^{i^{T}} z^{i}+\left(v-v^{i *}\right)^{T}\left(v-v^{i *}\right)\right]
$$

\footnotetext{
${ }^{4}$ The notation $\mathcal{S P}+\mathcal{T}$, with $\mathcal{S} \in \mathbb{R}^{n \times n}, \mathcal{T} \in \mathbb{R}^{n}$, and $\mathcal{P} \in \mathbb{R}^{n}$ denotes the set defined as $\left\{x \in \mathbb{R}^{n} \mid x=\mathcal{S} y+\mathcal{T}, y \in \mathcal{P}\right\}$.
} 


$$
\begin{gathered}
\mathcal{R}_{s}^{i}=\left\{\left(x^{i}, \dot{x}^{i}\right) \in \mathcal{X}_{a}^{i} \times \mathbb{R}^{n}: \dot{x}_{j}^{i} \leq \frac{1}{x_{j}^{i}+b_{2}}-\frac{1}{b_{2}} \text { if } x_{j}^{i} \geq-b_{2} \text { for all } j \in\{1,2, \ldots, n\}\right\} \\
\mathcal{R}_{h}^{i}=\left\{\begin{array}{l}
\left.\left(x^{i}, \dot{x}^{i}\right) \in \mathcal{X}_{a}^{i} \times \mathbb{R}^{n}: \exists j \in\{1,2, \ldots, n\} \text { such that } \dot{x}_{j}^{i}>\frac{1}{x_{j}^{i}+b_{2}}-\frac{1}{b_{2}} \text { and } x_{j}^{i} \geq-b_{2}\right\} \\
\text { and } \dot{x}_{k}^{i}<\frac{1}{x_{k}^{i}+b_{1}}-\frac{1}{b_{1}} \text { if } x_{k}^{i} \geq-b_{1} \text { for all } k \in\{1,2, \ldots, n\}
\end{array}\right\} \\
\mathcal{R}_{d}^{i}=\left\{\begin{array}{l}
\left(x^{i}, \dot{x}^{i}\right) \in \mathcal{X}_{a}^{i} \times \mathbb{R}^{n}: \exists j \in\{1,2, \ldots, n\} \text { such that } \dot{x}_{j}^{i} \geq \frac{1}{x_{j}^{i}+b_{1}}-\frac{1}{b_{1}} \text { and }-b_{1} \leq x_{j}^{i}<0 \\
\text { or } \exists j \in\{1,2, \ldots, n\} \text { such that } \dot{x}_{j}^{i}>\frac{1}{x_{j}^{i}+b_{1}}-\frac{1}{b_{1}} \text { and } x_{j}^{i}=0 \\
\text { or } \forall j \in\{1,2, \ldots, n\} \text { such that } x_{j}^{i}=\dot{x}_{j}^{i}=0
\end{array}\right\}
\end{gathered}
$$

and select $u_{c}^{i}$ such that $\dot{L}^{i}<0$ for all $z^{i} \neq 0$ and $v \neq v^{i *}$. One such selection is given by

$$
\begin{aligned}
u_{c}^{i}= & \mathcal{M}\left[\dot{v}^{i *}-\sum_{j=1}^{n} \frac{z_{j}^{i} e^{-z_{j}^{i}}}{x_{r_{j}}^{i}+\epsilon_{r_{j}}^{i}} s_{j}^{i T}-\alpha\left(v-v^{i *}\right)\right] \\
& +\mathcal{K} v+\mathcal{G S}^{i-1}\left[\left(x_{r}^{i}+\epsilon_{r}^{i}\right) e^{z^{i}}-\epsilon^{i}-\mathcal{T}\right],
\end{aligned}
$$

where $\alpha>0$ and

$$
v^{i *}=-\mathcal{S}^{i-1} \operatorname{diag}\left(z_{1}^{i}, \ldots, z_{n}^{i}\right)\left[x_{r_{1}}^{i}+\epsilon_{r_{1}}^{i}, \ldots, x_{r_{n}}^{i}+\epsilon_{r_{n}}^{i}\right]^{T} .
$$

\section{B. Shared Control Algorithm}

To design an s-control for the system (4) with constraints (5), recall that it is possible to define $N_{c}$ groups of $n$ constraints. To each group it is possible to associate one (and only one) c-controller. According to the definitions of $\mathcal{R}_{s}^{i}, \mathcal{R}_{h}^{i}$ and $\mathcal{R}_{d}^{i}$, given in Subsection III-A, let

$$
\begin{aligned}
& \mathcal{R}_{d}=\mathcal{R}_{d}^{1} \cup \mathcal{R}_{d}^{2} \cup \cdots \cup \mathcal{R}_{d}^{N_{c}}, \\
& \mathcal{R}_{h}=\mathcal{R}_{h}^{1} \cup \mathcal{R}_{h}^{2} \cup \cdots \cup \mathcal{R}_{h}^{N_{c}}, \\
& \mathcal{R}_{s}=\mathcal{R}_{s}^{1} \cup \mathcal{R}_{s}^{2} \cup \cdots \cup \mathcal{R}_{s}^{N_{c}} .
\end{aligned}
$$

Note that by the definitions of $\mathcal{R}_{s}^{i}$ and $\mathcal{R}_{d}^{i}, \mathcal{R}_{s}^{i} \cap \mathcal{R}_{d}^{i}=$ $\left\{\left(x^{i}, \dot{x}^{i}\right) \mid x^{i}=\dot{x}^{i}=0\right\}$.

Lemma 3: Consider the c-closed-loop system with the $i$-th c-control and $\alpha$ sufficiently large. The following statements hold.

(1) $\quad$ Suppose $x_{r}^{i}=0$. Then the point $\left(x^{i}, \dot{x}^{i}\right)=\mathcal{R}_{s}^{i} \cap \mathcal{R}_{d}^{i}$ is an equilibrium of the c-closed-loop system.

Suppose $x_{r}^{i} \neq 0$. Then the trajectory of the c-closed-loop system with the initial state $\left(x^{i}(0), \dot{x}^{i}(0)\right)=(0,0)$ remains in $\mathcal{R}$ and enters $\mathcal{R}_{s}^{i}$.

Note that the c-control (12) and the set division $\mathcal{R}_{d}^{i}, \mathcal{R}_{h}^{i}$ and $\mathcal{R}_{s}^{i}$ are given in terms of the variables $\left(x^{i}, \dot{x}^{i}\right)$. However, as remarked in Section III-A, there are several possible selections for the variable $x$. As a result, one has to translate them in the $(p, v)$ variables. This can be achieved as following.
Consider the regions $\mathcal{R}_{s}^{i}, \mathcal{R}_{h}^{i}$, and $\mathcal{R}_{d}^{i}$. Each one of these regions is associated, in a unique way, to a partion of the matrices $\mathcal{S}$ and $\mathcal{T}$, namely $\mathcal{S}^{i}$ and $\mathcal{T}^{i}$. By Lemma 2 all matrices $\mathcal{S}^{i}$ are invertible. Hence, it is possible to pull back the above sets in the space $\mathcal{P}_{a} \times \mathbb{R}^{n}$ using the relations ${ }^{5}$

$$
\begin{aligned}
\tilde{\mathcal{R}}_{s}^{i} & =\mathcal{S}_{i}^{e x}\left(\mathcal{R}_{s}^{i}-\mathcal{T}_{i}^{e x}\right), \\
\tilde{\mathcal{R}}_{h}^{i} & =\mathcal{S}_{i}^{e x}\left(\mathcal{R}_{h}^{i}-\mathcal{T}_{i}^{e x}\right), \\
\tilde{\mathcal{R}}_{d}^{i} & =\mathcal{S}_{i}^{e x}\left(\mathcal{R}_{d}^{i}-\mathcal{T}_{i}^{e x}\right),
\end{aligned}
$$

where $\mathcal{S}_{i}^{e x}=\operatorname{blockdiag}\left(\mathcal{S}^{i-1}, \mathcal{S}^{i-1}\right)$ and $\mathcal{T}_{i}^{e x}=$ $\operatorname{col}\left(\mathcal{T}^{i}, 0_{n}\right)$. Note that the sets $\tilde{\mathcal{R}}_{s}^{i}, \tilde{\mathcal{R}}_{h}^{i}$, and $\mathcal{R}_{d}^{i}$ have the following properties, which are inherited by properties of the sets $\mathcal{R}_{s}^{i}, \mathcal{R}_{h}^{i}$, and $\mathcal{R}_{d}^{i}$,

- $\tilde{\mathcal{R}}_{s}^{i} \cup \tilde{\mathcal{R}}_{h}^{i} \cup \tilde{\mathcal{R}}_{d}^{i}=\mathcal{R}$ for all $i \in\left\{1,2, \ldots, N_{c}\right\}$;

- $\tilde{\mathcal{R}}_{d}^{i} \cap \tilde{\mathcal{R}}_{d}^{j}=\emptyset$ for all $i, j \in\left\{1,2, \ldots, N_{c}\right\}$ and $i \neq j$;

- $\tilde{\mathcal{R}}_{s}^{i} \cap \tilde{\mathcal{R}}_{d}^{i}=\left\{(p, v) \mid \mathcal{S}_{i} p+\mathcal{T}_{i}=0, v=0\right\}$;

- $\tilde{\mathcal{R}}_{d}=\tilde{\mathcal{R}}_{d}^{1} \cup \cdots \cup \tilde{\mathcal{R}}_{d}^{N_{c}}, \tilde{\mathcal{R}}_{h}=\tilde{\mathcal{R}}_{h}^{1} \cup \cdots \cup \tilde{\mathcal{R}}_{h}^{N_{c}}, \tilde{\mathcal{R}}_{s}=$ $\tilde{\mathcal{R}}_{s}^{1} \cup \cdots \cup \tilde{\mathcal{R}}_{s}^{N_{c}}$.

On the basis of these sets, the sharing functions $k_{i}(p, v)$ are defined as

$$
k_{i}(p, v)= \begin{cases}1, & (p, v) \in \tilde{\mathcal{R}}_{s}^{i} \text { and }(p, v) \notin \tilde{\mathcal{R}}_{d}^{i}, \\ l_{i}(p, v), & (p, v) \in \tilde{\mathcal{R}}_{h}^{i}, \\ 0, & (p, v) \in \tilde{\mathcal{R}}_{d}^{i},\end{cases}
$$

where

$$
l_{i}(p . v)= \begin{cases}1, & \text { if }(p, v) \text { enters } \tilde{\mathcal{R}}_{h}^{i} \text { from } \tilde{\mathcal{R}}_{s}^{i}, \\ 0, & \text { if }(p, v) \text { enters } \tilde{\mathcal{R}}_{h}^{i} \text { from } \tilde{\mathcal{R}}_{d}^{i} .\end{cases}
$$

The c-controller is then given by the equation

$$
\begin{aligned}
u_{c}^{i}= & \mathcal{M}\left[\dot{v}^{i *}-\sum_{j=1}^{n} \frac{\log \frac{s_{j}^{i} p+t_{j}^{i}}{s_{j}^{i} p_{r}^{i}+t_{j}^{i}+\epsilon_{r_{j}}^{i}}}{s_{j}^{i} p+t_{j}^{i}} s_{j}^{i T}\right]+\mathcal{K} v \\
& -\alpha \mathcal{M}\left(v-v^{i *}\right)+\mathcal{G} p
\end{aligned}
$$

${ }^{5}$ Recall the notation in Footnote 4. In addition, the notation $\operatorname{col}(v, w)$ denotes a column vector obtained stacking the vectors $v$ and $w$ one above the other. 
where $\alpha>0$,

$$
v^{i *}=-\mathcal{S}^{i-1}\left[\begin{array}{c}
\left(s_{1}^{i} p_{r}+t_{1}^{i}+\epsilon_{r_{1}}^{i}\right) \log \frac{s_{1}^{i} p+t_{1}^{i}}{s_{1}^{i} p_{r}+t_{1}^{i}+\epsilon_{r_{1}}^{i}} \\
\vdots \\
\left(s_{n}^{i} p_{r}+t_{n}^{i}+\epsilon_{r_{n}}^{i}\right) \log \frac{s_{n}^{i} p+t_{n}^{i}}{s_{n}^{i} p_{r}+t_{n}^{i}+\epsilon_{r_{n}}^{i}}
\end{array}\right],
$$

and $p_{r}$ describes the desired behaviour of $p$.

Finally, the expression of the s-control in the $(p, v)$ coordinates is

$$
u_{s}(p, v)=\min _{i=1}^{N_{c}} k_{i}(p, v) u_{h}+\sum_{i=1}^{N_{c}}\left[\left(1-k_{i}(p, v)\right) u_{c}^{i}(p, v)\right]
$$

Note that $k_{i}$ is either 0 or 1 for all $i \in\left\{1,2, \ldots, N_{c}\right\}$ according to its definition given in (13). Therefore, $\underset{i=1}{N_{c}} k_{i}$ has only two values, 1 or 0 . In addition, by Lemma 2 , at any time instant no more than one c-control can be active: there is at most one $i$ such that $k_{i}=1$.

Lemma 4: Consider the system (4) in closed loop with the scontroller (15). Let $(p(t), v(t))$ be a trajectory of the system. Assume $(p(0), v(0)) \in \tilde{\mathcal{R}}_{s}$. Suppose there exists $\bar{t}>0$ such that $(p(\bar{t}), v(\bar{t})) \notin \tilde{\mathcal{R}}$. Then there exists a positive $\overline{\bar{t}}<\bar{t}$ such that $(p(\overline{\bar{t}}), v(\overline{\bar{t}})) \in \tilde{\mathcal{R}}_{d}$.

We are now ready to state the main result of the paper.

Theorem 1: Consider the system (4), the shared-control law (13), (14) and (15). Assume $p(0) \in \mathcal{P}_{a}$. Assume that the point $(\bar{p}, 0) \in \tilde{\mathcal{R}}_{s}$ is a globally asymptotically stable equilibrium for the h-closed-loop system. Then there exist $\alpha>0$ and $b_{2}>b_{1}>0$ such that the s-closed-loop system has the following properties.

(1) $p(t)$ stays in $\mathcal{P}_{a}$ for all $t \geq 0$.

(2) $\lim _{t \rightarrow \infty} p(t)=\bar{p}$.

(3) For all $t \geq 0$ such that $(p(t), v(t)) \in \tilde{\mathcal{R}}_{s} \backslash \tilde{\mathcal{R}}_{d}, u_{s}(t)=$ $u_{h}(t)$.

\section{Simulation Results}

In this section we discuss two simple case studies to illustrate the theoretical results. In the first one, a one degree-offreedom mechanical system with two inequality constraints in the position is considered, whereas the second example illustrates how the theory developed can be applied to mechanical systems subject to a set of constraints which is more general than those identified by the inequality (5).

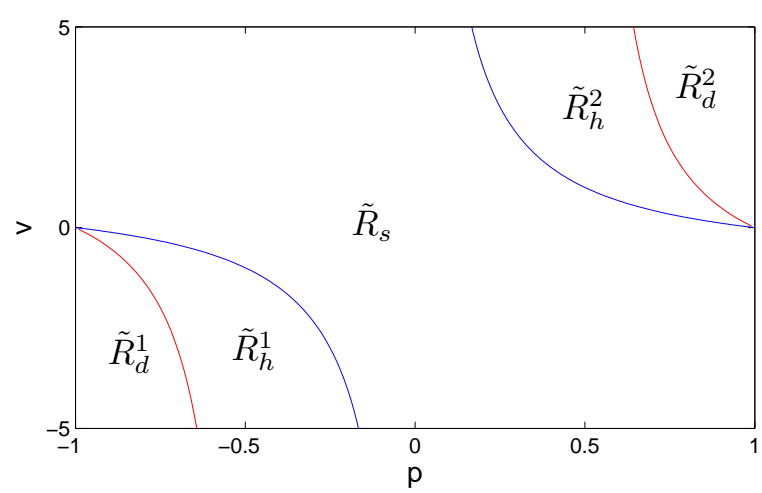

Fig. 1. Set Division $\left(b_{1}=0.5, b_{2}=1\right)$

\section{A. A One Degree-of-Freedom System with Bounded Position}

Consider a fully actuated, one degree-of-freedom, mechanical system described by the equations

$$
\begin{aligned}
& \dot{p}=v \\
& \dot{v}=-2 p-0.5 v+u
\end{aligned}
$$

Assume that $p$ has to track a desired trajectory $p_{d}$, subject to the constraints $p-1 \leq 0$ and $-p-1 \leq 0$. The set $\tilde{\mathcal{R}}_{s}, \tilde{\mathcal{R}}_{h}$ and $\tilde{\mathcal{R}}_{d}$ for this example, with $b_{1}=\overline{0.5}$ and $b_{2}=$ 1 , are depicted in Figure 1, which illustrates the properties discussed in Section III-B, namely $\tilde{\mathcal{R}}_{d}^{1} \cap \tilde{\mathcal{R}}_{d}^{2}=\emptyset, \mathcal{R}=$ $\tilde{\mathcal{R}}_{d} \cup \tilde{\mathcal{R}}_{h} \cup \tilde{\mathcal{R}}_{s}, \tilde{\mathcal{R}}_{d}^{1} \cap \tilde{\mathcal{R}}_{s}^{1}=\{(p, v)=(-1,0)\}, \tilde{\mathcal{R}}_{d}^{2} \cap \tilde{\mathcal{R}}_{s}^{2}=$ $\{(p, v)=(1,0)\}$.

Assume that $p_{d}$ is defined by the equations

$$
p_{d}(t)=\left\{\begin{aligned}
-1, & 0 \leq t<10 \\
0, & 10<t \leq 20 \\
1, & 20<t
\end{aligned}\right.
$$

Simulation results are displayed in Figure 2, which shows that the c-controller is active (i.e. $k=0$ ) only if $p$ is close to the boundary and the velocity in the direction of the boundary is above a certain value. In the first $10 s, k=0$ when $u_{c}^{1}$ is active, while in the last $10 s, k=0$ when $u_{c}^{2}$ is active. If $p$ is far away from the boundary, as the case for $t \in[10,20]$, none of the c-controllers is active and $k=1$ in this period.

\section{B. A Two Degree-of-Freedoms System with a Concave $\mathcal{P}_{a}$}

Consider a fully actuated, linear, mechanical system with two-degree-of freedoms described by the equations

$$
\begin{aligned}
& \dot{p}_{1}=v_{1}, \\
& \dot{p}_{2}=v_{2}, \\
& \dot{v}_{1}=-p_{1}-0.5 p_{2}-v_{1}-0.3 v_{2}+u_{1}, \\
& \dot{v}_{2}=-0.4 p_{1}-2 p_{2}-0.3 v_{1}+0.5 v_{2}+u_{2} .
\end{aligned}
$$

Assume that the feasible region $\mathcal{P}_{a}$ is defined by the conditions

$$
\begin{cases}p_{2} \geq 0 & \text { if } p_{1} \geq 0 \\ p_{1} \leq 0 & \text { if } p_{2} \leq 0\end{cases}
$$



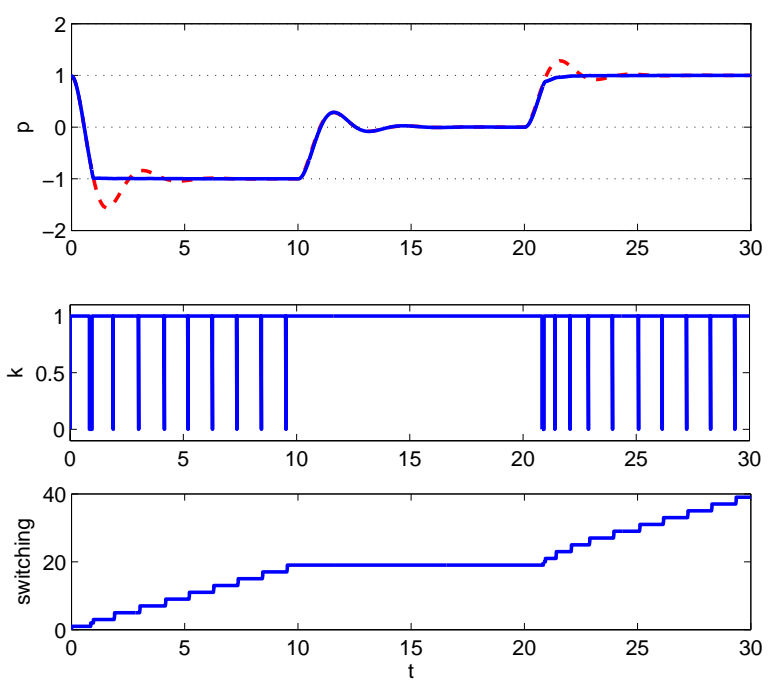

Fig. 2. Time histories of the variables $p$ (top) for the h-closed-loop system (red, dashed) and for the s-closed-loop system (blue, solid). Time history of $k$ (middle). Time history of switching times (bottom).

which yield a concave region. Using a variation of the construction in Section III, we have performed simulations with a shared control. Note that $\bar{p}=(0 ; 0)$ is at a "corner" of the admissible set. The simulation results are displayed in Figure 3 and shows that the trajectory of th shared closedloop system reaches the desired equilibrium without exiting the admissible set.

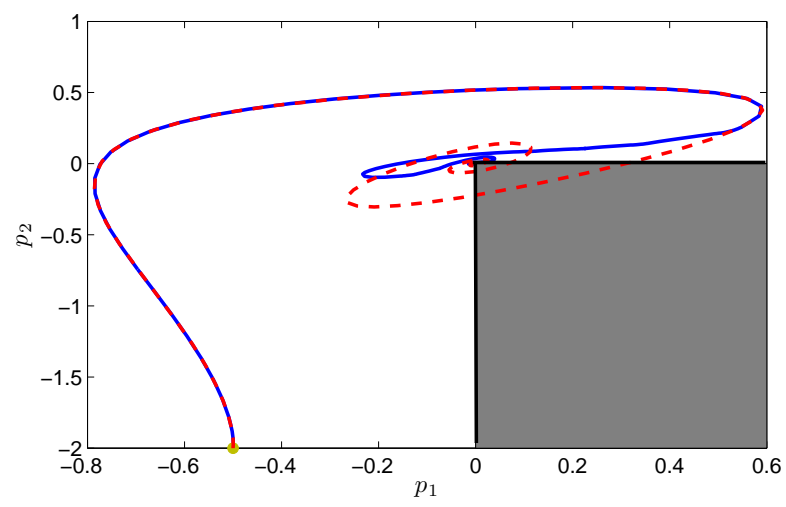

Fig. 3. Trajectories for the system (18): h-control (red, dashed) and scontrol (blue, solid).

\section{CONCLUSION}

We have developed a solution to a simple, abstract, shared control problem for a class of fully actuated linear mechanical systems. The shared control relies on the use of a hysteresis nonlinearity, which unites the h-controller and the c-controller and on the definition of safe and dangerous regions of operation. Theoretical properties of the closedloop system with the shared control are derived in the case in which the h-controller is designed for regulation. The theory is illustrated by two simple examples, the second of which highlights the applicability of the proposed ideas to a more general class of problems. Future work will be devoted to establishing properties of the s-closed-loop system in tracking problems and to design s-controllers for classes of nonlinear systems.

\section{REFERENCES}

[1] J.C.F. Winter and D. Dodou, "Preparing Drivers for Dangerous Situations", IEEE conference on Systems, Man and Cybernetics, pp.10501056, 2011

[2] P.Aigner and B.J.McCarragher, "Modeling and Constraining Human Interactions in Shared Control Utilizing a Discrete Event Framework", IEEE Transactions on Systems, Man and Cybernetics, vol.30, NO.3, pp. 369-379, May 2000.

[3] S.S. Nudehi, R. Mukherjee and M. Ghodoussi, "A Shared-Control Approach to Haptic Interface Design for Minimally Invasive Telesurgical Training", IEEE Transaction on Control Systems Technology, vol. 13, pp. 588-592, July 2005.

[4] H. Yu, M. Spenko and S. Dubowsky, "An Adaptive Shared Control System for an Intelligent Mobility Aid for the Elderly", Autonomous Robots, vol. 15, pp. 53-66, 2003.

[5] J. Borenstein and Y. Koren, "Real-Time Obstacle Avoidance for Fast Mobile Robots", IEEE Transactions on Systems, Man and Cybernetics, vol. 19, NO. 5, pp. 1179-1187, September/October 1989.

[6] Y. Koren and J. Borenstein, "Potential Field Methods and Their Inherent Limitations for Mobile Robot Navigation", in proceeding of IEEE on Robotics and Automation, pp. 1398-1404, April 1991.

[7] E. Gilbert and I. Kolmanovsky, "Nonlinear Tracking Control in the Presence of State and Control Constraints: a Generalized Reference Governor", Automatica, vol. 38, pp. 2063-2073, 2002.

[8] A. G. Wills and W. P. Heath, "Barrier Function Based Model Predictive Control", Automatica, vol. 40, pp. 1415-1422, 2004.

[9] L. Zaccarian, "On Dynamic Control Allocation for Input-Redundant Control Systems", in proceeding of IEEE on Decision and Control, pp. 1192-1197, December 12-14, 2007.

[10] ] M. Bodson, "Evaluation of Optimization Methods for Control Allocation", Journal of Guidance, Control and Dynamics, vol. 25, NO. 4, pp. 703-711, July-August 2002.

[11] C. Prieur and A. R. Teel, "Uniting Local and Global Output Feedback Controller", IEEE Transactions on Automatic Control, vol. 56, NO. 7 , pp. 1636-1679, July 2011.

[12] W. Ren and R. W. Beard, "Satisficing Approach to Human-in-the-Loop Safeguarded Control", in proceeding of American Control Conference, pp. 4985-4990, June 8-10, 2005.

[13] L. Vandeberghe and S. Boyd, "Semidefinite Programming", SIAM Review, vol. 38, NO. 1, pp. 49-95, 1996.

[14] C. Prieur, "Uniting Local and Global Controllers with Robustness to Vanishing Noise", Math. Control Signals Systems, vol.14, NO.2, pp.143-172, 2001.

[15] I. Kolmanovsky and G. Gilbert, "Theory and Computation of Disturbance Invariant Sets for Discrete-time Linear Systems", Mathematical Problems in Engineering, vol. 4, issue 4, pp. 317-367, 1998. 